\title{
Repeated sprint ability is not enhanced by caffeine, arginine, and branched-chain amino acids in moderately trained soccer players
}

\author{
Andrea Ermolao', Tobia Zanotto² ${ }^{2}$ Nicolò Carraro' ${ }^{1}$, Tommaso Fornasier' ${ }^{1}$ Marco Zaccaria', Daniel Neunhaeuserer', Marco Bergamin ${ }^{1, *}$ \\ ${ }^{1}$ Sport and Exercise Medicine Division, Department of Medicine, University of Padova, Padova, Italy \\ ${ }^{2}$ School of Health Sciences, Queen Margaret University, Edinburgh, United Kingdom
}

The aim was to investigate the effect of a dietary supplementation on the repeated sprint ability (RSA) performance in recreationally trained team sports athletes. Twelve young men underwent a RSA exercise protocol in five trials, in which participants ingested carbohydrates (CHO) plus caffeine (Caf), $\mathrm{CHO}$ plus arginine (Arg), $\mathrm{CHO}$ plus branchedchain amino acids (BCAA), CHO plus Caf, Arg, and BCAA (ALL), and $\mathrm{CHO}$ only. Heart rate, oxygen saturation, hematic lactate, ratings of per- ceived exertion, average sprint time, total time, best sprint time, peak power, and average power were taken. Data revealed no significant effects neither on physiological nor performance parameters with any of the supplements.

Keywords: Nutrition, Performance, Team sport, Dietary supplements

\section{INTRODUCTION}

The repeated sprint ability (RSA) consists of performing subsequent sprints with a short recovery time, and is typical for athletes engaged in team sports (Bishop et al., 2011). This type of performance involves both the anaerobic and aerobic energy systems, which is the typical metabolism of most team sports, for instance during soccer competitions (Spencer et al., 2004). Several factors are alleged to foster RSA performance, they include the energy supply capacities, such as phosphocreatine resynthesis, anaerobic glycolysis and aerobic metabolism, but also hydrogen ion $\left(\mathrm{H}_{+}\right)$ buffering and muscle activation (Girard et al., 2011). Furthermore, different exercise modalities can influence the magnitude of the RSA deterioration, indeed cycle ergometry produced lower performance (20\%-25\% decrease), compared to the 10\%-15\% reduction during running exercise (Fitzsimons et al., 1993).

Different training strategies can increase RSA (Bishop et al., 2011), but also optimal nutrition, including dietary supplemen- tation, may improve this performance. Caffeine (Caf), for instance, enhances endurance performance (Doherty and Smith, 2005) but also sprint ability (SA) on cycle-ergometry (Schneiker et al., 2006) and running (Glaister et al., 2008). Moreover, positive effects were detected on intermittent sprint testing, that is an exercise modality similar to the RSA, both on performance (Stuart et al., 2005), and rating of perceived exertion (RPE) (Cooper et al., 2014). Accordingly, the ergogenic effect of Caf on RSA was highlighted by Schneiker et al. (2006), in male team sport athletes during intermittent sprint testing and by Pontifex et al. (2010) in moderately trained male athletes.

Similarly to Caf, the use of branched-chain amino acids (BCAA) is well documented: on the whole, BCAA represent an energy source during intense exercise (Blomstrand, 2006), nevertheless, while the administration of BCAA combined with resistance training (RT) was confirmed to have positive effects on protein synthesis (Proud, 2009), the effects on endurance and sprint exercise remain controversial. In fact, BCAA do not seem to enhance this
${ }^{*}$ Corresponding author: Marco Bergamin (iD http://orcid.org/0000-0002-7441-4324 Sport and Exercise Medicine Division, Department of Medicine, University of Padova Via Giustiniani, 2-35128 Padova, Italy

Tel: +39-49-827-4429, Fax: +39-49-821-5862, E-mail: marco.bergamin@unipd.it Received: August 30, 2016 / Accepted: January 1, 2017
This is an Open Access article distributed under the terms of the Creative Commons Attribution Non-Commercial License (http://creativecommons.org/licenses/by-nc/4.0/) which permits unrestricted non-commercial use, distribution, and reproduction in any medium, provided the original work is properly cited. 
performance directly, but rather in ameliorating fatigue toleration (Greer et al., 2011; Meeusen et al., 2006). Evidences about the ergogenic effect of arginine (Arg) are controversial. Bailey et al. (2010) showed an improved oxygen consumption during progressive intensity exercise, demonstrating that Arg supplementation can be useful to increase aerobic capacity in untrained or moderately trained subjects. On the other hand, several investigations performed on healthy nonathlete subjects, did not report any benefits in cycle-ergometry performance (Liu et al., 2009), oxygen consumption during a treadmill test (Bescós et al., 2009; Sunderland et al., 2011) and metabolic (Liu et al., 2009), hormonal or cardio-respiratory responses (Forbes et al., 2013). In addition, results from clinical trials in highly trained athletes of judo (Liu et al., 2009; Tsai et al., 2009), tennis (Bescós et al., 2009), and cycling (Forbes et al., 2013) did not show any consistent improvement.

Overall, literature describes contrasting effects with regard to the use of $\mathrm{Caf}, \mathrm{BCAA}$, and Arg alone on exercise performance. For this reason, we aimed to compare these different components alone, combined, and versus a placebo beverage. The hypothesis is that the formulation including Caf, BCAA, and Arg leads to higher RSA performance than a single component or the placebo. To the best of our knowledge, this is the first study to examine the combined effect of a dietary supplementation formula including Caf, BCAA, and Arg on RSA performance in moderately trained soccer players.

\section{MATERIALS AND METHODS}

We recruited a group of 12 soccer players. Participants performed five trials in separate days that consisted of a battery of 11 subsequent sprints. Before each trial, subjects were administered $500 \mathrm{~mL}$ of dietary supplementation that consisted of the five treatment conditions (dietary supplements). Trials were performed in an indoor athletic field in order to standardize external conditions such as temperature, wind, humidity and ground characteristics, besides, all tests were carried out within one month and at the same time of the day.

\section{Participants}

Twelve young men were recruited through public announcement affixed to the Sport and Exercise Medicine Division of the Department of Medicine, University of Padova (Padova, Italy). The research protocol was performed in the same Institution. Information on the purpose and procedures of the study were given to each subject, and written consent was obtained before partici- pation. The study complied with the current laws of Italy for research on human participants and was examined and approved by the local review board.

Inclusion criteria for the participation to the study were: age between 20-30 years, being soccer players and with a training regimen of at least $4 \mathrm{hr}$ per week. Exclusion criteria included any history of central nervous dysfunction, or any transitorily conditions that limited sprinting performance or the range of motion. Additionally, subjects with clinical histories of severe cardiovascular disease that could limit or contraindicate exercise, angina or related symptoms, postural hypotension (defined as a fall in arterial blood pressure when changing position $>20 \mathrm{mmHg}$ in systole or $>10 \mathrm{mmHg}$ in diastole) were excluded. Furthermore, subjects were asked to abstain from consuming any nutritional or dietary supplements for the whole duration of the study. Additionally, the $48 \mathrm{hr}$ before any trial, participants were instructed to avoid intense or unaccustomed training sessions.

\section{Dietary supplementation}

The five treatment conditions were placebo $(\mathrm{P}), \mathrm{P}+\mathrm{Caf}(\mathrm{C}), \mathrm{P}+$ $\operatorname{Arg}(\mathrm{A}), \mathrm{P}+\mathrm{BCAA}(\mathrm{B}), \mathrm{P}+\mathrm{Caf}+\mathrm{Arg}+\mathrm{BCAA}(\mathrm{ALL}) . \mathrm{P}$ consisted of a mixture of carbohydrate (26.7 g), vitamins, mineral salts, and flavorings, and served as the base for each treatment condition. $\mathrm{C}$ was composed of $\mathrm{P}$ plus $300 \mathrm{mg}$ of $\mathrm{Caf}$, that is an average of 4 $\mathrm{mg} / \mathrm{kg}$, which is considered an optimal threshold for caffeine efficacy (Goldstein et al., 2010). A was comprised of P plus $3 \mathrm{~g}$ of Arg, while B was P plus $5 \mathrm{~g}$ of BCAA. Finally, ALL represented the combination of $\mathrm{P}$ with the addition of $300 \mathrm{mg}$ of $\mathrm{Caf}, 3 \mathrm{~g}$ of Arg and $5 \mathrm{~g}$ of BCAA. The volume of the five beverages was 500 $\mathrm{mL}$ and the osmolarity was kept constant for each condition to mask the presence of different substances.

\section{Performance measures}

Photocell gates (Alge-Timing, Lustenau, Austria) were placed in correspondence of the initial and final lines of the track, and subjects were required to start the sprint $1.5 \mathrm{~m}$ behind the starting line. Heart rate (HR) was registered with a Polar s810i wearable device (Polar Electro, Kempele, Finland), Oxygen saturation (OS) was measured by pulse oximetry (OxiMax NPB-40, Nellcor, Canada), finally, a capillary lactate analyzer (Lactate plus, Nova Biomedical, Waltham, MA, USA) was adopted to measure the hematic concentration of lactate (HL).

\section{Experimental procedure}

Subjects performed 5 testing sessions in 5 different days, held 
approximately 1 week apart. One hr before each session, subjects consumed, with a random assignment, one of the dietary supplementations prepared in a black opaque water bottle. Both participants and assessors were blinded. Ten minutes before each testing sessions, subjects underwent a standardized warm-up consisting of $5 \mathrm{~min}$ of jogging, five sprints at moderate intensity and $2 \mathrm{~min}$ of jogging. The RSA protocol consisted of 11 maximal sprints interspersed with brief recoveries of $20 \mathrm{sec}$ along a $20-\mathrm{m}$ track (Marella and Risaliti, 1999). During the recovery period, participants were instructed to return to the starting line within 20 sec.

Standardized verbal encouragement was provided throughout the sprint to minimize possible bias effects due to lack of motivation and to promote a maximal effort (McNair, 1996). Physiological measures, HR, OS, and HL, were measured at baseline and after exercise, within a few seconds from the end of the last sprint, while, RPE was collected only immediately after the eleventh sprint of each trial.

Following physical performance parameters were determined: average sprint time (AST), total time (TT), peak power (PP), and average power (AP). PP and AP were calculated with the formula $P=\left(0.5 \mathrm{M} \mathrm{L}^{\wedge} 2\right) \bullet \mathrm{T}-3[\mathrm{~W}]$, whereas $\mathrm{M}$ is body mass, $\mathrm{L}$ is distance of run and $\mathrm{T}$ is time. Both PP and AP were also expressed for body mass (W/kg) and respectively named peak power expressed for body mass (BMPP) and average power expressed for body mass (BMAP).

\section{Statistical analysis}

Statistical analysis was carried out using IBM SPSS ver. 18.0 (IBM Co., Armonk, NY, USA). Results are expressed as means \pm standard deviation (SD) or percentage (\%). The Shapiro-Wilk test was carried out to check if data were normally distributed, in ad-

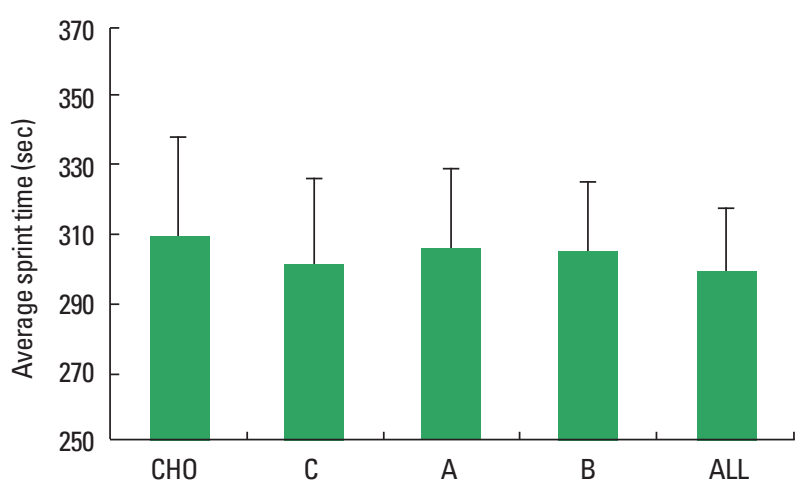

Fig. 1. Primary physical performance outcomes: average sprint time. $\mathrm{CHO}$, carbohydrates only (placebo); $C$, carbohydrates plus caffeine; $A$, carbohydrates plus arginine; $B$, carbohydrates plus branched chain amino acids; All, carbohydrate plus caffeine, arginine and branched chain amino acids. dition, Mauchly test was used to test whether the variances of the differences between conditions were equal. All dependent variables were analyzed through analysis of variance (ANOVA) for repeated measures. Following post hoc tests were performed with Bonferroni correction. For RPE, a Friedman 2-way ANOVA was computed, due to a nonnormal data distribution. Significance limits were set at alpha level of $P=0.05$ ( $80 \%$ power).

\section{RESULTS}

Three out of the 12 participants dropped out from the study due to sport injuries unrelated with the experimental protocol, thus final analysis was performed exclusively on the results of the nine subjects who completed the investigation. The study population was comprised of nine male athletes, aged between 21 and 27 years-old (mean \pm SD, 22.6 \pm 2.1 years); an average volume of training of $7.9 \pm 3.9 \mathrm{hr}$ per week was found. Mean height and weight were $182.3 \pm 8.4 \mathrm{~cm}$, and $74.5 \pm 5.4 \mathrm{~kg}$, respectively (body mass index, $22.5 \pm 1.7 \mathrm{~kg} / \mathrm{m}^{2}$ ). Baseline evaluation of the physiological parameters revealed a mean HR of $68 \pm 11.6 \mathrm{bpm}$, mean OS of $99.4 \% \pm 0.8 \%$, and mean HL of $1.11 \pm 0.4 \mathrm{mmol} / \mathrm{L}$.

Mauchly test failed on AST and TT outcomes, hence Greinhouse-Geisser correction was used to correct for violations of sphericity. The analysis showed no differences among the types of beverage in both AST $(F=3.033 ; P=0.071)$ and TT $(F=2.670 ; P=$ 0.86 ) (Figs. 1, 2). Similarly within subject analysis did not detect any statistical difference in the BMAP, as well as for postexercise OS, HL, and RPE (Table 1).

On the other hand, $\mathrm{PP}(F=3.508 ; P=0.017)$ and $\mathrm{AP}(F=2.670$; $P=0.04)$ as well as postexercise $\operatorname{HR}(F=3.011 ; P=0.032)$ and

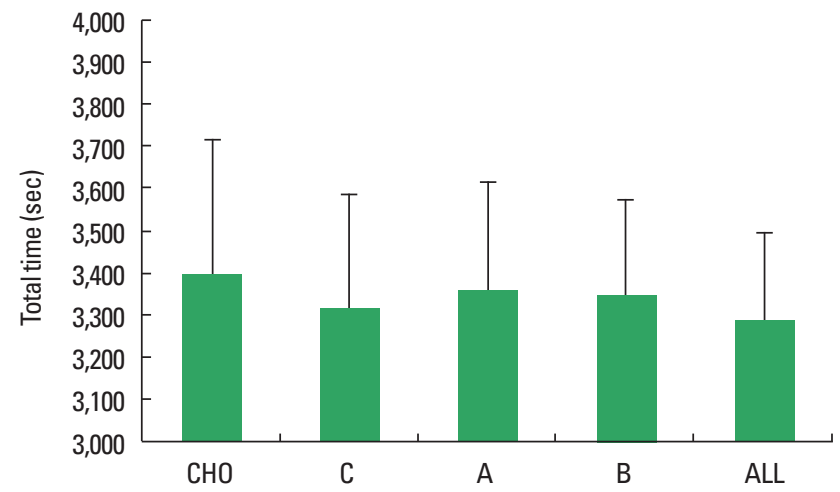

Fig. 2. Primary physical performance outcomes: total time. CHO, carbohydrates only (placebo); $C$, carbohydrates plus caffeine; $A$, carbohydrates plus arginine; $B$, carbohydrates plus branched chain amino acids; All, carbohydrate plus caffeine, arginine, and branched chain amino acids. 
Table 1. Physiological and performance outcomes

\begin{tabular}{|c|c|c|c|c|c|c|c|c|}
\hline & $\begin{array}{c}\text { Body mass } \\
\text { average power } \\
(\mathrm{W} / \mathrm{kg})\end{array}$ & $\begin{array}{c}\text { Postexercise } \\
\text { hematic lactate } \\
(\mathrm{mmol} / \mathrm{L})\end{array}$ & $\begin{array}{c}\text { Postexercise } \\
\text { oxygen saturation } \\
(\%)\end{array}$ & $\begin{array}{l}\text { Rating of } \\
\text { perceived effort } \\
\text { (RPE) }\end{array}$ & $\begin{array}{l}\text { Peak power } \\
\text { (W) }\end{array}$ & $\begin{array}{c}\text { Average power } \\
\text { (W) }\end{array}$ & $\begin{array}{l}\text { Postexercise heart } \\
\text { rate (bpm) }\end{array}$ & $\begin{array}{c}\text { Body mass peak } \\
\text { power (W/kg) }\end{array}$ \\
\hline$P$ & $7.12 \pm 1.78$ & $6.20 \pm 3.18$ & $96.22 \pm 1.30$ & $13.66 \pm 1.65$ & $596.11 \pm 133.82$ & $530.60 \pm 138.47$ & $167.22 \pm 9.97$ & $8.01 \pm 1.76$ \\
\hline C & $7.55 \pm 1.66$ & $7.18 \pm 3.26$ & $96.55 \pm 2.12$ & $13.55 \pm 1.13$ & $638.33 \pm 147.12$ & $564.17 \pm 135.87$ & $156.44 \pm 14.12$ & $8.56 \pm 1.94$ \\
\hline$A$ & $7.26 \pm 1.57$ & $6.45 \pm 2.40$ & $97.22 \pm 0.66$ & $13.55 \pm 0.72$ & $623.23 \pm 129.96$ & $542.45 \pm 128.46$ & $162.88 \pm 14.72$ & $8.37 \pm 1.72$ \\
\hline B & $7.26 \pm 1.37$ & $5.92 \pm 2.01$ & $96.44 \pm 1.13$ & $13.33 \pm 1.41$ & $604.13 \pm 102.68$ & $540.23 \pm 95.72$ & $163.11 \pm 13.86$ & $8.12 \pm 1.48$ \\
\hline All & $7.66 \pm 1.35$ & $8.05 \pm 2.41$ & $96.55 \pm 1.66$ & $13.66 \pm 1.22$ & $655.97 \pm 126.15$ & $572.91 \pm 115.23$ & $165.22 \pm 16.17$ & $8.78 \pm 1.54$ \\
\hline
\end{tabular}

Values are presented as mean \pm standard deviation.

P, carbohydrate only; C, carbohydrates plus caffeine; A, carbohydrates plus arginine; B, carbohydrates plus branched chain amino acids; All, carbohydrate plus caffeine, arginine, and branched chain amino acids.

Within group analyses did not found any statistically significant change after the consumption of each nutritional supplement.

Table 2. Physiological and performance outcomes according to the within subject analysis (PP, AP, HR, BMPP)

\begin{tabular}{llccc}
\hline & Peak power $(\mathrm{W})$ & Average power $(\mathrm{W})$ & Postexercise heart rate $(\mathrm{bpm})$ & Body mass peak power $(\mathrm{W} / \mathrm{kg})$ \\
\hline P & $596.11 \pm 133.82$ & $530.60 \pm 138.47$ & $167.22 \pm 9.97$ & $8.01 \pm 1.76$ \\
C & $638.33 \pm 147.12$ & $564.17 \pm 135.87$ & $156.44 \pm 14.12$ & $8.56 \pm 1.94$ \\
A & $623.23 \pm 129.96$ & $542.45 \pm 128.46$ & $162.88 \pm 14.72$ & $8.37 \pm 1.72$ \\
B & $604.13 \pm 102.68$ & $540.23 \pm 95.72$ & $163.11 \pm 13.86$ & $8.12 \pm 1.48$ \\
All & $655.97 \pm 126.15$ & $572.91 \pm 115.23$ & $165.22 \pm 16.17$ & $8.78 \pm 1.54$ \\
\hline
\end{tabular}

Values are presented as mean \pm standard deviation.

This table highlights the physiological and performance outcomes that resulted in significant difference, after the consumption of supplements, according to the Within Subject Analysis (PP, AP, HR, BMPP).

P, carbohydrate only; C, carbohydrates plus caffeine; A, carbohydrates plus arginine; B, carbohydrates plus branched chain amino acids; All, carbohydrate plus caffeine, arginine and branched chain amino acids; PP, peak power; AP, average sprint time; HR, heart rate, BMPP, body mass peak power.

$\operatorname{BMPP}(F=3.307 ; P=0.022)$ showed statistical significant difference when performances were carried out under different conditions. Nevertheless, although ANOVA-Repeated Measures showed a statistical significance, the magnitude of the difference, in these variable, was not so large to determine a statistically difference in any of the pairwise comparisons. Results are reported in Table 2.

\section{DISCUSSION}

The aim of this study was to evaluate the acute effects of ingesting Caf, Arg, and BCAA on RSA performance, both in single or combined conditions, to assess a potential synergistic effect of these substances. The results of this experiment suggest that adding Caf, Arg, and BCAA to a $\mathrm{CHO}$ beverage does not improve RSA in soccer players, indeed, the consumption of these substances did not significantly improve physical performance, neither in single modality, nor when administered together, compared to the placebo condition.

A reasonable explanation for the lack of effects retrieved may rely on the adoption of a $\mathrm{CHO}$ drink as the base for each treatment condition, including placebo: $\mathrm{CHO}$ is an essential nutrient for sprint performance (American College of Sports et al., 2000) and its consumption, along with the other substances, may have masked the extent of decrease in performance that could have occurred with a noncaloric placebo. Anyhow, the use of a common $\mathrm{CHO}$ base was opted in order to evaluate the possible ergogenic effects of supplements beyond the well-established contribution from $\mathrm{CHO}$ consumption. Furthermore, the selection of the exercise protocol might play a key role in detecting the deterioration of RSA. In this study, the "Coverciano" test was chosen because of the affinity with competition-like conditions in team sports (Marella and Risaliti, 1999), however, its short duration may have reduced the expected decline in performance, and we can speculate that adding subsequent sprint sets could extend this gap, allowing the potential effects of these substances to counteract fatigue towards the last stages of exercise.

Among the supplements we investigated, Caf can be potentially considered as the most influent in term of improving of the SA (Pontifex et al., 2010; Schneiker et al., 2006). Possible mechanisms for enhanced performance after Caf ingestion can include the stimulation of the sympathetic nervous system (Goldstein et al., 2010), the increased heart rate, higher blood perfusion to skel- 
etal muscles and hepatic gluconeogenesis (Hartley et al., 2004). A few studies investigating the catecholaminergic response to high intensity exercises advanced the hypothesis that caffeine might foster physical performance by increased adrenaline secretion, that would augment the glycolitic activity (Doherty et al., 2002; Stuart et al., 2005). Notwithstanding, in the same studies the higher adrenaline bio-availability did not always reflect better performance, as in our experiment, the administration of caffeine alone did not produce significant results whereas the more conservative Bonferroni post hoc analysis method was utilized.

The contribution of BCAA to exercise performance is ascribed to the capability of serving as an energy source: their oxidation produces alanine that is a central precursor of hepatic gluconeogenesis. Precisely, they represent the first source of aminoacids during intense exercise (Blomstrand, 2006). In the current study, BCAA ingestion did not result in better performance, in fact, the RSA protocol might have been relatively short in duration for this mechanism to occur to a sufficient extent. Despite the controversy around the ergogenic effect of Arg, as described in the introduction, previous research outlined that Arg supplementation can enhance the onset of oxygen consumption (Koppo et al., 2009), that could enclose the oxygenation deficit occurring with physical exercise and therefore reducing lactate accumulation (Jones and Poole, 2005).

Results from our study evidenced that postexercise HL differed between $C$ and ALL conditions, anyhow, these changes were not statistically significant. In literature, the ingestion of caffeine was related with an augmented $\mathrm{HL}$ at the end of endurance exercise (Anderson and Hickey, 1994; Chesley et al., 1998; Graham and Spriet, 1995), nevertheless, studies investigating high intensity exercises report equivocal results; some of them underlined increased HL (Bell et al., 2001; Crowe et al., 2006; Doherty et al., 2002; Schneiker et al., 2006), while others did not acquaint any variations (Doherty, 1998; Doherty et al., 2002; Greer et al., 1998). Interestingly, the majority of the studies reporting increased $\mathrm{HL}$ also detected improved performance, independently from training status of participants, as it is confirmed by our results.

It has recently been suggested that the accumulation of lactate and other metabolic byproducts could be diminished by the production of nitric oxide (Camic et al., 2010). Arg might play a role in this mechanism, even if there is still lack of evidence about its capability of modifying blood flow in healthy human beings. A few studies found postexercise metabolites unaltered after Arg consumption, compared to placebo (Liu et al., 2009; Tsai et al., 2009), which is in accord to the weak response of HL measured after Arg administration in the current experiment.

The remaining postexercise physiological parameters, HR and OS, did not show any relevant trendline between treatments. RPEs associated with BCAA showed a mild reduction tendency, despite nonsignificant, in agreement with previous research on fatigue toleration (Greer et al., 2011; Meeusen et al., 2006; Strüder et al., 1998).

In conclusion, it should be acknowledged that an inversion of tendency in mean sprint times was ascertained for each treatment condition in the last sprints. This phenomena could be explained on the basis of a psychological and motivational incentive arising from the termination of the trial; some authors suggested that performance is influenced by athletes' consciousness of the duration of the protocol (Billaut et al., 2011).

\section{Study limitations}

The sample size represents a structural limit of this study. The low number of participants is a resultant of the experimental design selection; an acute intervention design was preferred to limit the confounding variables such as physical fitness of participants, type of nutrition throughout the study and environmental factors. Moreover, the study design resulted in optimal compliance of subjects and served as a precompetition simulation, that was the objective of the research topic. Lastly, it must be acknowledged that other nutritional supplements could have been examined for the study, foremost creatine, whose ergogenic potential in physical performance is widely investigated, though, its acute effects on performance are controversial (Deutekom et al., 2000). Besides, creatine-caffeine coingestion was described to counteract the ergogenic effect of caffeine (Lee et al., 2011).

In summary, the nutritional supplement designed for this study, composed of Caf, Arg, and BCAA, did not enhance RSA performance compared to the placebo condition. Despite a slight improvement trend line was retrieved after the ingestion of the experimental supplement, data analysis revealed no statistical differences in any performance parameters taken for the audit. Null effects were found on RSA whether the substances were administered in single modality or all together: a synergistic effect does not seem to exist for these substances. Although nutritional supplements containing carbohydrate, Caf, Arg, and BCAA are currently available on the market and amply advertised for team sports, the results of this study do not support the hypothesis that adding Caf, Arg, and BCAA to a carbohydrate drink is more beneficial to RSA than ingesting carbohydrate alone in recreationally trained young men. Future perspectives for consolidating the re- 
trieved evidences concern the enlargement of sample size and the extension of exercise duration. The slight decrement of performance throughout the entire protocol suggest that prolonging the duration of exercise by adding sprint sets to the original protocol would increase the efficacy in testing the effects of nutritional aids on RSA.

\section{CONFLICT OF INTEREST}

No potential conflict of interest relevant to this article was reported.

\section{REFERENCES}

American College of Sports Medicine; American Dietetic Association; Dietitians of Canada. Joint Position Statement: nutrition and athletic performance. American College of Sports Medicine, American Dietetic Association, and Dietitians of Canada. Med Sci Sports Exerc 2000;32: 2130-2145.

Anderson DE, Hickey MS. Effects of caffeine on the metabolic and catecholamine responses to exercise in 5 and 28 degrees C. Med Sci Sports Exerc 1994;26:453-458.

Bailey SJ, Winyard PG, Vanhatalo A, Blackwell JR, DiMenna FJ, Wilkerson DP, Jones AM. Acute L-arginine supplementation reduces the $\mathrm{O} 2$ cost of moderate-intensity exercise and enhances high-intensity exercise tolerance. J Appl Physiol (1985) 2010;109:1394-1403.

Bell DG, Jacobs I, Ellerington K. Effect of caffeine and ephedrine ingestion on anaerobic exercise performance. Med Sci Sports Exerc 2001;33: 1399-1403.

Bescós R, Gonzalez-Haro C, Pujol P, Drobnic F, Alonso E, Santolaria ML, Ruiz O, Esteve M, Galilea P. Effects of dietary L-arginine intake on cardiorespiratory and metabolic adaptation in athletes. Int J Sport Nutr Exerc Metab 2009;19:355-365.

Billaut F, Bishop DJ, Schaerz S, Noakes TD. Influence of knowledge of sprint number on pacing during repeated-sprint exercise. Med Sci Sports Exerc 2011;43:665-672.

Bishop D, Girard O, Mendez-Villanueva A. Repeated-sprint ability - part II: recommendations for training. Sports Med 2011;41:741-756.

Blomstrand E. A role for branched-chain amino acids in reducing central fatigue. J Nutr 2006;136:544S-547S.

Camic CL, Housh TJ, Mielke M, Zuniga JM, Hendrix CR, Johnson GO, Schmidt RJ, Housh DJ. The effects of 4 weeks of an arginine-based supplement on the gas exchange threshold and peak oxygen uptake. Appl Physiol Nutr Metab 2010;35:286-293.

Chesley A, Howlett RA, Heigenhauser GJ, Hultman E, Spriet LL. Regula- tion of muscle glycogenolytic flux during intense aerobic exercise after caffeine ingestion. Am J Physiol 1998;275(2 Pt 2):R596-603.

Cooper R, Naclerio F, Allgrove J, Larumbe-Zabala E. Effects of a carbohydrate and caffeine gel on intermittent sprint performance in recreationally trained males. Eur J Sport Sci 2014;14:353-361.

Crowe MJ, Leicht AS, Spinks WL. Physiological and cognitive responses to caffeine during repeated, high-intensity exercise. Int J Sport Nutr Exerc Metab 2006;16:528-544.

Deutekom M, Beltman JG, de Ruiter CJ, de Koning JJ, de Haan A. No acute effects of short-term creatine supplementation on muscle properties and sprint performance. Eur J Appl Physiol 2000;82:223-229.

Doherty M. The effects of caffeine on the maximal accumulated oxygen deficit and short-term running performance. Int J Sport Nutr 1998;8: 95-104.

Doherty M, Smith PM. Effects of caffeine ingestion on rating of perceived exertion during and after exercise: a meta-analysis. Scand J Med Sci Sports 2005;15:69-78.

Doherty M, Smith PM, Davison RC, Hughes MG. Caffeine is ergogenic after supplementation of oral creatine monohydrate. Med Sci Sports Exerc 2002;34:1785-1792

Fitzsimons M, Dawson B, Ward D, Wilkinson A. Cycling and running tests of repeated sprint ability. Aust J Sci Med Sport 1993;25:82-87.

Forbes SC, Harber V, Bell GJ. The acute effects of L-arginine on hormonal and metabolic responses during submaximal exercise in trained cyclists. Int J Sport Nutr Exerc Metab 2013;23:369-377.

Girard O, Mendez-Villanueva A, Bishop D. Repeated-sprint ability - part I: factors contributing to fatigue. Sports Med 2011;41:673-694.

Glaister M, Howatson G, Abraham CS, Lockey RA, Goodwin JE, Foley P, McInnes G. Caffeine supplementation and multiple sprint running performance. Med Sci Sports Exerc 2008;40:1835-1840.

Goldstein ER, Ziegenfuss T, Kalman D, Kreider R, Campbell B, Wilborn C, Taylor L, Willoughby D, Stout J, Graves BS, Wildman R, Ivy JL, Spano M, Smith AE, Antonio J. International society of sports nutrition position stand: caffeine and performance. J Int Soc Sports Nutr 2010;7:5.

Graham TE, Spriet LL. Metabolic, catecholamine, and exercise performance responses to various doses of caffeine. J Appl Physiol (1985) 1995;78:867-874.

Greer BK, White JP, Arguello EM, Haymes EM. Branched-chain amino acid supplementation lowers perceived exertion but does not affect performance in untrained males. J Strength Cond Res 2011;25:539-544.

Greer F, McLean C, Graham TE. Caffeine, performance, and metabolism during repeated Wingate exercise tests. J Appl Physiol (1985) 1998;85: 1502-1508.

Hartley TR, Lovallo WR, Whitsett TL. Cardiovascular effects of caffeine 
in men and women. Am J Cardiol 2004;93:1022-1026.

Jones AM, Poole DC. Oxygen uptake kinetics in sport, exercise and medicine. New York: Routledge; 2005.

Koppo K, Taes YE, Pottier A, Boone J, Bouckaert J, Derave W. Dietary arginine supplementation speeds pulmonary $\mathrm{VO} 2$ kinetics during cycle exercise. Med Sci Sports Exerc 2009;41:1626-1632.

Lee CL, Lin JC, Cheng CF. Effect of caffeine ingestion after creatine supplementation on intermittent high-intensity sprint performance. Eur J Appl Physiol 2011;111:1669-1677.

Liu TH, Wu CL, Chiang CW, Lo YW, Tseng HF, Chang CK. No effect of short-term arginine supplementation on nitric oxide production, metabolism and performance in intermittent exercise in athletes. J Nutr Biochem 2009;20:462-468.

Marella M, Risaliti M. Il libro dei test: le prove di valutazione fisica per tutti gli sport. Milano: Edizioni Correre; 1999.

McNair PJ. Verbal encouragement of voluntary muscle action: reply to commentary by Roger Eston. Br J Sports Med 1996;30:365.

Meeusen R, Watson P, Dvorak J. The brain and fatigue: new opportunities for nutritional interventions? J Sports Sci 2006;24:773-782.

Pontifex KJ, Wallman KE, Dawson BT, Goodman C. Effects of caffeine on repeated sprint ability, reactive agility time, sleep and next day performance. J Sports Med Phys Fitness 2010;50:455-464.
Proud CG. mTORC1 signalling and mRNA translation. Biochem Soc Trans 2009;37(Pt 1):227-231.

Schneiker KT, Bishop D, Dawson B, Hackett LP. Effects of caffeine on prolonged intermittent-sprint ability in team-sport athletes. Med Sci Sports Exerc 2006;38:578-585.

Spencer M, Lawrence S, Rechichi C, Bishop D, Dawson B, Goodman C. Time-motion analysis of elite field hockey, with special reference to repeated-sprint activity. J Sports Sci 2004;22:843-850.

Strüder HK, Hollmann W, Platen P, Donike M, Gotzmann A, Weber K. Influence of paroxetine, branched-chain amino acids and tyrosine on neuroendocrine system responses and fatigue in humans. Horm Metab Res 1998;30:188-194.

Stuart GR, Hopkins WG, Cook C, Cairns SP. Multiple effects of caffeine on simulated high-intensity team-sport performance. Med Sci Sports Exerc 2005;37:1998-2005.

Sunderland KL, Greer F, Morales J. VO2max and ventilatory threshold of trained cyclists are not affected by 28-day L-arginine supplementation. J Strength Cond Res 2011;25:833-837.

Tsai PH, Tang TK, Juang CL, Chen KW, Chi CA, Hsu MC. Effects of arginine supplementation on post-exercise metabolic responses. Chin J Physiol 2009;52:136-142. 